\title{
The Role of FLT3-ITD and CEBPA Mutations on Prognosis of Acute Lymphoblastic Leukaemia in Turkish patients
}

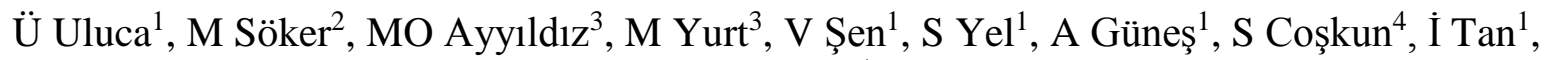 \\ C Şahin ${ }^{1}$
}

\begin{abstract}
Introduction: Acute lymphoblastic leukaemia (ALL) is the most common malignancy in childhood. Although some prognostic factors have been defined to date, estimation of prognosis is currently not perfect. Previous studies have shown an association of FLT3 with poor prognosis and CCAAT/enhancer binding protein $\alpha$ (CEBPA) mutation with the development of acute myeloid leukaemia (AML). Here we aimed to evaluate the prognostic value of FLT3-ITD and CEBPA mutations in ALL.

Materials and Method: Sixty-one patients with ALL were included in the study. Patients were divided into three risk groups according to BFM risk classification. All of the patients were examined for FLT3-ITD mutations and 45 of them for CEBPA mutations. Mutation positive and negative patients were compared in terms of their risk groups, translocations and cell lineage. The clinical courses of the patients were appraised.
\end{abstract}

Results: FLT3-ITD mutation was detected in three of 61 patients, and CEBPA mutations were detected in 11 of 45 patients. The incidence of established prognostic indicators including BFM risk classification, $\mathrm{t}(9 ; 22)$;BCR-ABL, $\mathrm{t}(1 ; 19)$;E2A-PBX1, $\mathrm{t}(12 ; 21)$;TELAML1, $\mathrm{t}(4 ; 11)$;MLL-AF4 were similar between FLT3-ITD and CEBPA positive and negative patients. A patient with an FLT3-ITD mutation was very susceptible to pancytopenia after maintenance treatment and another two patients with FLT3-ITD mutations were more prone to febrile neutropenia.

Conclusion: Our results suggest that CEBPA or FLT3-ITD mutations may not be related to ALL prognosis in Turkish patients. However, FLT3-ITD mutation may have an influence on the response of bone marrow to chemotherapy.

Keywords: Acute lymphoblastic leukaemia, CEBPA, FLT3, mutation, prognosis

From: ${ }^{1}$ Department of Pediatrics, ${ }^{2}$ Department of Pediatric Hematology, ${ }^{3}$ Department of Hematology-Oncology and ${ }^{4}$ Department of Medical Genetics, Dicle University, Medical School, Diyarbakir, Turkey

Correspondence: Dr Ünal Uluca, Dicle University Medical School, Department of Pediatrics, Diyarbakır, Turkey. Phone: +90 4122488001 / 5787, Fax: +90 4122488440.

E-mail: ulucaunal@hotmail.com 


\section{INTRODUCTION}

Although the genetic background of oncogenesis is not fully understood, deterioration in cell differentiation is a crucial component for the development of cancer. (1). Acute lymphoblastic leukaemia (ALL), which comprises of B-cell lineage and T-cell lineage subgroup cancers is the most common malignancy in childhood cancers (2). Risk classification of the disease may lead to different outcome estimates.

Numerous prognostic factors related to the decision of treatment regimens have been determined in pediatric ALL. However, there are disagreements among physicians about the use of these prognostic factors for ALL (3). Due to these differences, it may be difficult to compare results of different clinical studies. Thus, new reliable risk classification and prognostic factors are required.

Although some prognostic factors were determined for ALL such as age, gender, white blood count (WBC) at diagnosis of ALL, response to treatment, DNA index, $\mathrm{t}$ (9:22), and $\mathrm{t}$ (4:11), there is a need for more specific markers (3).

FMS-like tyrosine kinase-3 (FLT3) is a class 3 tyrosine kinase family receptor that is expressed in hematopoietic cells related to lymphocyte development. These receptors play important roles in hematopoiesis, especially on lymphoid lineage (4). Internal tandem duplication (ITD) of FLT3 gene led to disruption of FLT3 auto inhibition. Previous studies have suggested that FLT3 mutations and their expression may be associated with poor prognosis for acute myeloid leukaemia (AML) and overexpression of FLT3 receptor protein has been shown in AML and B-cell acute lymphoblastic leukaemia (B-ALL). It has been considered that FLT3 inhibitor therapy may benefit since overexpression of the FLT3 gene has been shown in leukaemia $(4,5)$. Therefore, showing FLT3 mutations may be beneficial 
for both treatment and prognosis of acute leukaemia. However, in ALL the association between FLT3 and prognosis of disease is unclear and further investigates are required.

CCAAT/enhancer binding protein $\alpha$ (CEBPA), a transcription factor, is expressed in granulocytes, monocytes and eosinophils. When CEBPA is bound to DNA, it activates transcription of two N-terminal trans-activation domains (6). Mutations of this transcription factor may be related to prognosis of some malignancies such as AML and ALL. CCAAT/enhancer binding protein $\alpha$, expression is reduced or inhibited during heterozygous or homozygous mutation for some polymorphisms of the CEBPA gene. Reduction or inhibition of CEBPA expression has been found to be related to the development of AML.

However, the prognostic role of CEBPA in AML is controversial (6-8). On the other hand, CEBPA mutation is suggested to be related to the survival of patients with ALL (9). However, few studies have been conducted for the evaluation of the pathogenetic and/or prognostic role of the CEBPA mutation in $\operatorname{ALL}(6,7,9-11)$.

In this study, we aimed to investigate whether FLT3-ITD or CEBPA mutations are related with the established prognostic factors in children with ALL.

\section{MATERIALS AND METHOD}

The study included 61 children (44 male, 17 female) with ALL, who were diagnosed and treated between February 2009 and March 2014. Age, gender, white blood count (WBC), hemoglobin value and platelet count at diagnosis, treatment protocol, and risk groups of patients were recorded. Patients were classified as standard risk group (SRG), medium risk group (MRG) and high risk group (HRG) according to BFM (Berlin-Frankfurt-Munster) risk classification. Standard risk group included patients aged 1-6 years who had white blood cell 
counts of less than $20000 / \mathrm{mm}^{3}$ at diagnosis, good response to prednisone and no-T-ALL. The high risk group included those with a poor prednisone response, and/or evidence of $\mathrm{t}(9 ; 22) ; \mathrm{BCR} / \mathrm{ABL}$, and/or evidence of $\mathrm{t}(4 ; 11) ; \mathrm{MLL} / \mathrm{AF} 4$. Patients with no HRG or SRG criteria were accepted as MRG. Flow cytometry and oncogene fusion transcript screening for $\mathrm{t}(9 ; 22)$;BCR-ABL, $\mathrm{t}(1 ; 19) ; \mathrm{E} 2 \mathrm{~A}-\mathrm{PBX} 1, \mathrm{t}(12 ; 21)$;TEL-AML1, and $\mathrm{t}(4 ; 11) ;$ MLL-AF4 were recorded. FLT3-ITD and FLT3-D835 mutations were studied in all patients. CEBPA mutations were studied in 45 of the patients. The study protocol was approved by the local Ethics Committee.

Cells from the diagnostic bone marrow samples were cultured for 24 hours according to standard cytogenetic protocols. The chromosomes were banded using a GTG technique and karyotyping was performed. All patients were confirmed by conventional cytogenetic and reverse transcriptase polymerase chain reaction (RT-PCR) for $t(9 ; 22)$; BCR-ABL, $\mathrm{t}(1 ; 19)$;E2A-PBX1, t(12;21);TEL-AML1 and t(4;11);MLL-AF4 fusion transcript.

\section{DNA extractions and genotyping}

Genomic DNA was extracted from bone marrow cells using a DNA isolation kit (High Pure PCR Template Preparation-Roche, Mannheim, Germany) according to the manufacturer's instructions. The DNA concentration was determined using a Nano-Drop spectrophotometer (Thermo Scientific, USA) and samples were stored at $-20^{\circ} \mathrm{C}$ until polymerase chain reaction (PCR) was performed.

Screening for CEBPA mutations were performed using a high resolution melting curve based on LightCycler 480 assay (Roche Diagnostics, Mannheim, Germany).

Analysis for FLT3/D835 mutations was carried out by LightCycler based
high resolution melting curve analysis with the forward primer 5'-


AGAGACCTGGCCGCCAGGA-3'; reverse primer 5'-CAACACAAAATAGCCGTATAA3’and hybridization probes, 835V GGATTGGCTCGAGATATCATG -FL and Red LC640 TGATTCCAACTATGTTGTCAGGGGCAATGT-PH. The PCR reaction was performed in a $20 \mu 1$ reaction volume contained with $5 \mu \mathrm{L}$ DNA, $1 \mu \mathrm{l}(0.2 \mu \mathrm{M})$ each of forward and reverse primer, $1 \mu$ hybridization probes, $1,6 \mu \mathrm{l}(3 \mathrm{mM}) \mathrm{MgCl} 2$ and $2 \mu \mathrm{L}$ LightCycler-FastStart DNA Master HybProbe Mix (Roche Diagnostics, Mannheim, Germany).

Amplification was performed with 45 cycles using $55{ }^{\circ} \mathrm{C}$ annealing temperature. Final melting curve analysis was started at $40{ }^{\circ} \mathrm{C}$ up to $95{ }^{\circ} \mathrm{C}$ with slope of $0.2{ }^{\circ} \mathrm{C} / \mathrm{s}$ and continuous detection with channel 640. Data were analysed using the LightCycler 480 software (Roche Diagnostics, USA) and the second derivative maximum method by high resolution melting curve analysis mode.

Detection of FLT3/ITD, polymerase chain reaction was performed using specific primers, 11F， 5'-GCAGCCTCACATTGCCCC-3' 12R. 5'-CTTCAGCATTTTGACGGCAACC-3' and probes 20F, 5'-CCGCCAGGAACGTGCTTG-3'; 20R, 5'-GCAGCCTCACATTGCCCC3'. Amplification was performed in a $20 \mu 1$ reaction volume with $5 \mu 1 \mathrm{DNA}, 0.3 \mu \mathrm{M}$ each of primers and probes, $3 \mathrm{mM} \mathrm{MgCl} 2$, and $5 \mu \mathrm{L}$ LightCycler-FastStart Master Mix (Roche Diagnostics, Mannheim, Germany). Denaturing, annealing, and extension steps were performed at $95{ }^{\circ} \mathrm{C}$ for $5 \mathrm{~s}, 64{ }^{\circ} \mathrm{C}$ for $10 \mathrm{~s}, 72{ }^{\circ} \mathrm{C}$ for $15 \mathrm{~s}$, respectively for 45 cycles. Final melting curve analysis was started at $40{ }^{\circ} \mathrm{C}$ up to $95{ }^{\circ} \mathrm{C}$ with slope of $0.2{ }^{\circ} \mathrm{C} / \mathrm{s}$.

Sequence variants in the DNA, either homozygous or heterozygous, gave rise to heteroduplexes that were easily detected by high-resolution melting curve analysis from earlier melting products. 


\section{Statistical analysis}

Numerical variables were shown as mean \pm standard deviation. The comparison of the groups having abnormally distributed numerical data was performed with Mann-Whitney U test or Kruskal-Wallis test. Chi-squared test was used to compare categorical variables between independent groups. A p-value less than 0.05 was accepted as statistically significant.

\section{RESULTS}

A total of 61 children (44 male, 17 female) with ALL were evaluated. The mean age of the patients at diagnosis was $6.1 \pm 3.8$ years. In complete blood count analysis at diagnosis: white blood cell count (WBC) was $34400 \pm 60208 / \mathrm{mm}^{3}$, hemoglobin was $8.7 \pm 2.2 \mathrm{~g} / \mathrm{dL}$, and the platelet count was $109559 \pm 97080 / \mathrm{mm}^{3}$. The characteristics of patients in each risk groups were summarized in Table 1.

Table 1. Characteristics of risk groups at diagnosis (mean \pm standard deviation or number)

\begin{tabular}{lllll}
\hline & $\begin{array}{l}\text { SRG } \\
(\mathbf{n = 1 7 )}\end{array}$ & $\begin{array}{l}\text { MRG } \\
(\mathbf{n = 3 7 )}\end{array}$ & $\begin{array}{l}\text { HRG } \\
(\mathbf{n}=\mathbf{7})\end{array}$ & $\boldsymbol{p}$ \\
\hline Diagnosis age & $3.5 \pm 1.6$ & $6.6 \pm 3.8$ & $9.5 \pm 4.7$ & $<0.001^{*}, 0.003^{\dagger}$, \\
& & & & $0.0001^{\ddagger}, 0.12^{\S}$ \\
Gender (M/F) & $12 / 5$ & $25 / 12$ & $7 /-$ & $0.21^{*}$ \\
Positive FLT3-ITD & - & 2 & 1 & $0.33^{*}$ \\
Positive CEBPA & 3 & 6 & 2 & $0.86^{*}$ \\
WBC, /mm & $6622 \pm 3686$ & $46093 \pm 70572$ & $49747 \pm 52876$ & $0.01^{*}$ \\
Hemoglobin, g/dL & $9.1 \pm 1.8$ & $8.2 \pm 2.4$ & $10.9 \pm 2.2$ & $0.16^{*}$ \\
Platelet count, /mm & $93707 \pm 99970$ & $113866 \pm 100297$ & $1127585 \pm 70709$ & $0.60^{*}$ \\
\hline
\end{tabular}

SRG: standard risk group, MRG: medium risk group, HRG: high risk group, *between three groups, ${ }^{\dagger}$ : between SRG and MRG,

‡: between SRG and HRG, ${ }^{\S}$ : between MRG and HRG 
Thirty four patients had been treated according to TR ALL BFM 2000 treatment protocol and 27 patients had been treated according to ALLIC BFM 2009 treatment protocol.

FLT3-ITD mutation was detected in $3(4.9 \%)$ of all patients and CEBPA mutation in 11 (24.4\%). The characteristics of mutation positive patients are summarized in Table 2.

Table 2. Characteristics of FLT3-ITD and CEBPA mutations positive patients

\begin{tabular}{|c|c|c|c|c|c|c|c|c|}
\hline & $\begin{array}{l}\text { Diagnosis age, } \\
\text { years }\end{array}$ & $\begin{array}{l}\text { WBC } \\
\left(/ \mathbf{m m}^{3}\right) \text { at } \\
\text { diagnosis }\end{array}$ & $\mathbf{t}(\mathbf{1 2 , 2 1 )}$ & $\mathbf{t}(\mathbf{1 , 1 9 )}$ & $\mathbf{t}(\mathbf{9 , 2 2})$ & $t(4,11)$ & $\begin{array}{l}\text { Cell } \\
\text { lineage }\end{array}$ & $\begin{array}{l}\text { BFM } \\
\text { Risk } \\
\text { group }\end{array}$ \\
\hline \multicolumn{9}{|c|}{ Patients with FLT3-ITD mutation } \\
\hline Patient 1 & 13,5 & 158000 & - & - & - & - & B cell & $\mathrm{HRG}$ \\
\hline Patient 2 & 5 & 3980 & - & - & - & - & $\mathrm{T}$ cell & MRG \\
\hline Patient 3 & 7,5 & 30100 & + & - & - & - & $\mathrm{B}$ cell & MRG \\
\hline \multicolumn{9}{|c|}{ Patients with CEBPA mutation } \\
\hline Patient 4 & 8 & 5790 & - & - & - & - & B cell & $\mathrm{MRG}$ \\
\hline Patient 5 & 14.5 & 50700 & - & - & - & - & $\mathrm{T}$ cell & $\mathrm{HRG}$ \\
\hline Patient 6 & 7 & 7550 & - & - & - & - & $\mathrm{T}$ cell & MRG \\
\hline Patient 7 & 3.5 & 6950 & - & + & - & - & B cell & SRG \\
\hline Patient 8 & 4 & 11900 & - & - & - & - & $\mathrm{T}$ cell & MRG \\
\hline Patient 9 & 1,5 & 3980 & - & - & - & - & B cell & SRG \\
\hline Patient 10 & 1 & 14100 & - & - & - & - & B cell & MRG \\
\hline Patient 11 & 3 & 76800 & + & - & - & - & B cell & MRG \\
\hline Patient 12 & 5 & 12400 & - & - & - & - & B cell & SRG \\
\hline Patient 13 & 7 & 33200 & - & - & - & - & B cell & MRG \\
\hline Patient 14 & 12.5 & 57850 & - & - & - & - & B cell & HRG \\
\hline
\end{tabular}

SRG: standard risk group, MRG: medium risk group, HRG: high risk group

FLT3-D835 mutation was not detected in any patient. There was no significant difference between the BFM risk groups in terms of the presence of FLT3-ITD and CEBPA mutations ( $p=0.33$ and $p=0.86$, respectively). There were no differences between FLT3ITD positive and negative groups in terms of positivity or negativity of $t(9 ; 22)$;BCR-ABL1, $\mathrm{t}(1 ; 19) ; \mathrm{E} 2 \mathrm{~A}-\mathrm{PBX} 1, \quad \mathrm{t}(12 ; 21) ; \mathrm{ETV} 6-\mathrm{RUNX} 1 \quad$ and $\mathrm{t}(4 ; 11) ;$ MLL-AF4 translocations. 
Alike, these translocations were observed in similar frequency among CEBPA mutation positive and negative patients. In respect to B-cell or T-cell distribution, positive-negative FLT3 mutation groups and positive-negative CEBPA mutation groups were found to be similar $(p=0.71, p=0.52)$. There were no differences between mutation positivity when patients were divided into two age subgroups [1-6 years age group and $>6$ years group] $(\mathrm{p}=0.42)$. Similarly, there were no differences between positive and negative mutations when patients were divided into two groups according to their ages [1-6 years age group and $>6$ years age group] $(p=0.67)$.

One patient had developed a relapse. This patient did not have FLT3-ITD or CEBPA mutations. Bone marrow transplantation was applied to a patient with CEBPA mutation in the high risk group. No significant differences were found when B-cell ALL and T-cell ALL patients were compared in terms of WBC, platelet count and hemoglobin values at diagnosis, $(p=0.27, p=0.37$ and $p=0.27$, respectively).

Pancytopenia developed in a patient with medium risk having an FLT3-ITD mutation after maintenance treatment. Thus, $25 \%$ of the total dose of methotrexate and 6-mercaptopurine was given to this patient. On the other hand, another two patients with FLT3-ITD mutations were frequently hospitalized due to febrile neutropenia. No patients died.

\section{DISCUSSION}

Recently, ALL patients have had better survival rates because of effective treatment regimens and improved healthcare following chemotherapy. In order to increase survival rates of high risk patients, reliable markers for risk estimation and better treatment modalities are required.

Internal tandem duplication of FLT3 in juxtamembrane domain is a mutation detected frequently in AML but is rarely seen in ALL (12). Previous studies have found increased 
expression of FLT3 RNA and FLT3-ITD proteins in the bone marrow of AML and ALL patients compared with healthy subjects. FLT3-ITD mutation is associated with overexpression of FLT3-ITD protein. FLT3 expression may be related to survival or proliferation of leukaemic blast cells $(4,12)$. FLT3-ITD mutation is frequently observed in AML patients and it is related to poor prognosis in AML. The prognostic role of FLT3-ITD mutation in ALL is unclear and this mutation is rarely found in ALL $(12,13)$. Thus, the role of FLT3-ITD mutation in ALL is controversial and further investigations are required. In addition, detecting the relationship between ALL prognosis and FLT3 mutation may indicate the beneficial effects of kinase inhibitors in ALL management.

In this study, FLT3-ITD mutation distribution was found comparable among three ALL risk groups and not related with other known prognostic factors such as WBC and age at diagnosis and translocations of $(9,22),(4,11)$ or $(12,21)$. Therefore, our results showed that FLT3-ITD mutation may not be related to ALL prognosis in children. Because of the limited number of patients, our results need to be confirmed by further studies with more patients. Two FLT3-ITD mutation positive patients developed neutropenia and pancytopenia developed in another patient with FLT3-ITD mutation. The explanation of this predisposition is unclear. However, FLT3-ITD mutation may aggravate leukopenia as a side effect of chemotherapy.

It has been stated that the CEBP gene family plays an important role in proliferation and differentiation. In proliferation and differentiation of normal lymphocytes and lymphoid malignancies, the functions of the CEBP genes remain largely unknown. CEBP mutations are accepted to be associated with a relatively good overall prognosis (14). In a study performed by Park et al. CCAAT/enhancer binding protein $\alpha$ mutated patients had better prognoses than CEBPA wild-type patients in AML (8). On the other hand, loss of CEBP expression is 
thought to be concomitant with B-cell differentiation (14). CCAAT/enhancer binding protein $\alpha$, genes are stated to be novel oncogenes in B cell precursor ALL (14). Thus, CEBP genes may be related to the pathogenesis and prognosis of ALL. However, data about the prognostic role of the CEBPA mutation in ALL is limited.

The present study found that CEBPA mutation was not related with other known favorable or unfavorable prognostic factor in ALL such as WBC at diagnosis, $(9,22),(4,11)$, $(12,21)$ translocations, age at diagnosis and B-cell or T-cell subgroups. These results suggest that CEBPA may not be related to ALL prognosis.

The main limitations of this study were the small number of patients and the absence of a control group.

In conclusion, we did not detect any relationship between mutation positive and negative patients with FLT3-ITD or CEBPA mutations in terms of known prognostic factors and clinical course of patients. However FLT3-ITD mutation may affect the response to chemotherapy. 


\section{REFERENCES}

1. Koschmieder S, Halmos B, Levantini E, Tenen DG. Dysregulation of the C/EBPalpha differentiation pathway in human cancer. J Clin Oncol 2009; 27: 619-28.

2. Zhang J, Ding L, Holmfeldt L, Wu G, Heatley SL, Payne-Turner D et al. The genetic basis of early T-cell precursor acute lymphoblastic leukaemia. Nature 2012; 481: $157-$ 163.

3. Smith M, Arthur D, Camitta B, Carroll AJ, Crist W, Gaynon P et al. Uniform approach to risk classification and treatment assignment for children with acute lymphoblastic leukemia. J Clin Oncol. 1996; 14: 18-24.

4. Vora HH, Shukla SN, Brahambhatt BV, Mehta SH, Patel NA, Parikh SK et al. Clinical relevance of FLT3 receptor protein expression in Indian patients with acute leukemia. Asia Pac J Clin Oncol 2010; 6: 306-19.

5. Meshinchi S, Stirewalt DL, Alonzo TA, Boggon TJ, Gerbing RB, Rocnik JL et al. Structural and numerical variation of FLT3/ITD in pediatric AML. Blood 2008; 111: 4930-933.

6. Paz-Priel I, Friedman A. C/EBPalpha dysregulation in AML and ALL. Crit Rev Oncog 2011; 16: 93-102.

7. Chiaretti S, Messina M, Tavolaro S, Zardo G, Elia L, Vitale A et al. Gene expression profiling identifies a subset of adult T-cell acute lymphoblastic leukemia with myeloid-like gene features and over-expression of miR-223. Haematologica 2010; 95: $1114-21$.

8. Park SH, Chi HS, Cho YU, Jang S, Park CJ. CEBPA single mutation can be a possible favorable prognostic indicator in NPM1 and FLT3-ITD wild-type acute myeloid leukemia patients with intermediate cytogenetic risk. Leuk Res 2013; 37: 1488-94. 
9. Lautner-Csorba O, Gézsi A, Semsei AF, Antal P, Erdélyi DJ, Schermann G et al. Candidate gene association study in pediatric acute lymphoblastic leukemia evaluated by Bayesian network based Bayesian multilevel analysis of relevance. BMC Med Genomics 2012; 5: 42.

10. Figueroa ME, Wouters BJ, Skrabanek L, Glass J, Li Y, Erpelinck-Verschueren CA et al. Genome-wide epigenetic analysis delineates a biologically distinct immature acute leukemia with myeloid/T-lymphoid features. Blood 2009; 113: 2795-2804.

11. Chapiro E, Russell L, Radford-Weiss I, Bastard C, Lessard M, Struski S et al. Overexpression of CEBPA resulting from the translocation $t(14 ; 19)(q 32 ; q 13)$ of human precursor B acute lymphoblastic leukemia. Blood 2006; 108: 3560-63.

12. Gilliland DG, Griffin JD. The roles of FLT3 in hematopoiesis and leukemia. Blood 2002; 100: 1532-42.

13. Taketani T, Taki T, Sugita K, Furuichi Y, Ishii E, Hanada R et al. FLT3 mutations in the activation loop of tyrosine kinase domain are frequently found in infant ALL with MLL rearrangements and pediatric ALL with hyperdiploidy. Blood 2004; 103: 1085 1088.

14. Akasaka T, Balasas T, Russell LJ, Sugimoto KJ, Majid A, Walewska R et al. Five members of the CEBP transcription factor family are targeted by recurrent IGH translocations in B-cell precursor acute lymphoblastic leukemia (BCP-ALL). Blood 2007; 109: 3451-461. 